\title{
Dynamics of current-driven phase-slip centers in superconducting strips
}

\author{
G. Berdiyorov,,${ }^{1,2, *}$ K. Harrabi, ${ }^{2}$ F. Oktasendra, ${ }^{2}$ K. Gasmi, ${ }^{2}$ A. I. Mansour, ${ }^{2}$ J. P. Maneval, ${ }^{3}$ and F. M. Peeters ${ }^{4}$ \\ ${ }^{1}$ Department of Physics, Loughborough University, Leicestershire LE11 3TU, United Kingdom \\ ${ }^{2}$ Physics Department, King Fahd University of Petroleum and Minerals, 31261 Dhahran, Saudi Arabia \\ ${ }^{3}$ Laboratoire Pierre Aigrain, ENS 24 rue Lhomond, Paris F-75231, France \\ ${ }^{4}$ Departement Fysica, Universiteit Antwerpen, Groenenborgerlaan 171, B-2020 Antwerpen, Belgium
}

(Received 19 June 2014; revised manuscript received 25 July 2014; published 8 August 2014)

\begin{abstract}
Phase-slip centers/lines and hot spots are the main mechanisms for dissipation in current-carrying superconducting thin films. The pulsed-current method has recently been shown to be an effective tool in studying the dynamics of phase-slip centers and their evolution to hot spots. We use the time-dependent Ginzburg-Landau theory in the study of the dynamics of the superconducting condensate in superconducting strips under external current and zero external magnetic field. We show that both the flux-flow state (i.e., slow-moving vortices) and the phase-slip line state (i.e., fast-moving vortices) are dynamically stable dissipative units with temperature smaller than the critical one, whereas hot spots, which are localized normal regions where the local temperature exceeds the critical value, expand in time, resulting ultimately in a complete destruction of the condensate. The response time of the system to abrupt switching on of the overcritical current decreases with increasing both the value of the current (at all temperatures) and temperature (for a given value of the applied current). Our results are in good qualitative agreement with experiments we have conducted on $\mathrm{Nb}$ thin strips.
\end{abstract}

DOI: 10.1103/PhysRevB.90.054506

PACS number(s): 74.20.De, 74.25.Ha

\section{INTRODUCTION}

It is known that the resistive state of current-carrying (quasi-)one-dimensional superconductors is characterized by phase-slip centers (PSCs), where oscillations of the order parameter allow the phase to relax by quanta of $2 \pi[1,2]$. PSCs result in the formation of pronounced steps in both voltage vs temperature [3] and current-voltage characteristics [4,5] of narrow superconducting strips with transverse dimensions of the order of the superconducting coherence length $\xi$. For superconducting samples with lateral dimensions larger than $\xi$, PSCs can be generalized to phase-slip lines with almost uniform suppression of the order parameter along the sample [6]. The oscillations of the order parameter may also occur in the form of propagating waves carrying the zero of the order parameter across the sample. Such waves (named kinematic vortices) have been discovered in numerical simulations [7,8] and experimental evidence for the existence of such kinematic vortices was reported in Ref. [9]. These vortices move with velocity [9] $v_{k v} \approx 10^{5} \mathrm{~m} / \mathrm{s}$, which is much larger than the maximal measured speed of Abrikosov vortices [10] $v_{a v} \approx 10^{3} \mathrm{~m} / \mathrm{s}$. Because of their very high velocity kinematic vortices do not retain their circular structure $[7,8]$, leading to a rearrangement of the vortex lattice in the system [11].

Another mechanism for the resistive state of currentcarrying superconductors is the formation of hot spots (HSs) [12], where the local temperature exceeds the critical value, suppressing the superconducting condensate completely. It is believed that the PSC regime is confined to a small temperature range in the vicinity of the transition temperature $T_{c}[3,5]$ and that the HS mechanism dominates at lower temperatures [12]. However, recent studies implementing the pulsed-current technique revealed that hot spots never form unless PSCs have first been nucleated [13-16].

\footnotetext{
*Present address: Qatar Environment and Energy Research Institute, Doha, Qatar.
}

This experimental technique has been shown to be an effective tool in the study of the evolution of the resistive state in current-driven superconductors and furthermore it has a number of advantages over conventional techniques such as multiprobe voltage measurements [17], laser imaging [9], and radio-frequency synchronization $[5,18]$.

In this work we study the dynamics of the superconducting condensate in superconducting strips under abrupt switching on of dc current using the time-dependent Ginzburg-Landau (TDGL) theory. We find that the resistive state in such current-carrying superconducting samples is characterized by either dynamically stable (slow or fast) moving vortices or expanding HS depending on the external condition (e.g., temperature and current). The flux-flow state (i.e., slow motion of vortices) and the phase-slip state (i.e., fast-moving vortices) are characterized by a saturating output voltage, whereas the HS state results in a monotonic increase of the output signal as a function of time. We confirm recent experimental findings [13] that the phase-slip state is always proceeded by the HS state. The response time of the superconducting condensate to the applied current pulse is studied as a function of temperature, the applied current, and the sample size.

\section{THEORETICAL FORMALISM}

As model system we consider a superconducting strip with computational unit cell of lateral dimensions $L$ and $w$. The thickness $d$ is much smaller than the coherence length $\xi$ and the penetration depth $\lambda$ (see Fig. 1). Current is applied along the $x$ direction. For this system we solved numerically the following TDGL equations in the zero electric potential gauge [19]:

$$
\begin{gathered}
\frac{u}{\sqrt{1+\gamma^{2}|\psi|^{2}}}\left(\frac{\partial}{\partial t}+\frac{\gamma^{2}}{2} \frac{\partial|\psi|^{2}}{\partial t}\right) \psi \\
=(\nabla-i \mathbf{A})^{2} \psi+\left(1-|\psi|^{2}\right) \psi, \\
\frac{\partial \mathbf{A}}{\partial t}=\operatorname{Re}\left[\psi^{*}(-i \boldsymbol{\nabla}-\mathbf{A}) \psi\right]-\kappa^{2} \operatorname{rot} \operatorname{rot} \mathbf{A} .
\end{gathered}
$$




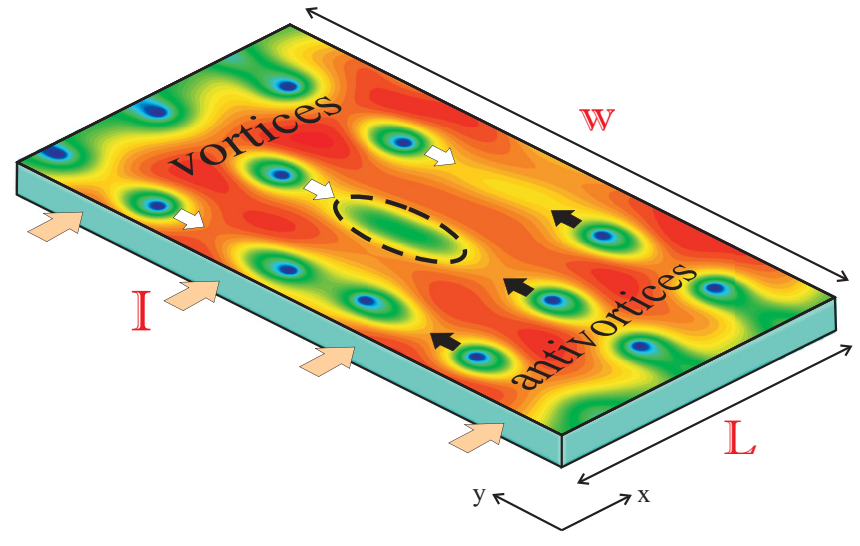

FIG. 1. (Color online) The model system: a superconducting slab (width $w$ and with periodic boundary condition in the $x$ direction) under applied dc current $I$. A contour plot of the superconducting condensate is superimposed on top of the sample with the direction of moving vortices/antivortices indicated by white/black arrows.

In these equations, we express the length in units of the coherence length $\xi$ and the vector potential is scaled to $\Phi_{0} /(2 \pi \xi)$ (where $\Phi_{0}$ is the magnetic flux quantum). Time is in units of the Ginzburg-Landau relaxation time $t_{0}=4 \pi \lambda^{2} / c^{2} \rho_{n}$ ( $\rho_{n}$ is the normal-state resistivity), the voltage is in units of $V_{0}=c \Phi_{0} \rho_{n} / 8 \pi^{2} \lambda \xi$, and the current density is measured in $j_{0}=c \Phi_{0} / 8 \pi^{2} \lambda^{2} \xi$. The parameters $u$ and $\gamma$, which are a measure of the different relaxation times, are taken as $u=5.79$ and $\gamma=10$ [19]. The numerical results are obtained for $\kappa=10$. The infinite strip is implemented through periodic boundary conditions $\psi(x, y)=\psi(x, y+L)$ and $\mathbf{A}(x, y)=$ $\mathbf{A}(x, y+L)$ in the $y$ direction. The superconducting-vacuum boundary condition $\left.(\boldsymbol{\nabla}-\boldsymbol{i} \mathbf{A}) \psi\right|_{x=0, w}$ is used in the $x$ direction. Current is applied by taking the following boundary condition for the vector potential in the $x$ direction, $\left.\operatorname{rot} \mathbf{A}\right|_{z}(x=$ $0, w)=H \pm H_{I}$, where $H$ is the applied magnetic field (zero in our case) and $H_{I}=2 \pi I / c$ is the magnetic field induced by the current $I$. Note that in Eqs. (1) and (2) the screening of the magnetic field is neglected which is valid for the samples with width $w$ much smaller than the Pearl length $\Lambda=2 \lambda / d$ or for samples infinite in the $z$ direction. These coupled nonlinear differential equations are solved self-consistently in a zero electrostatic potential gauge [20], which was recently shown to be very effective in studying the dynamics of superconducting vortices [11]. We also conducted simulations where we couple the TDGL equations with the heat transfer equation (see Ref. [21] for the details of the numerical approach) and found that heating effects due to the moving vortices result only in quantitative changes in our findings. The temperature is indirectly included in the calculations through $\xi, \lambda$, and $H_{c 2}$, whose temperature dependencies are given by $\xi(T)=\xi(0) / \sqrt{1-T / T_{c}}, \lambda(T)=$ $\lambda(0) / \sqrt{1-T / T_{c}}$, and $H_{c 2}(T)=H_{c 2}(0)\left(1-T / T_{c}\right)$, respectively, where $T_{c}$ is the superconducting transition temperature.

\section{I- $V$ CHARACTERISTICS}

As a representative example, we consider a superconducting strip with period $L=1 \mu \mathrm{m}$ for different values of the width $w$. Zero-temperature coherence length is taken to be
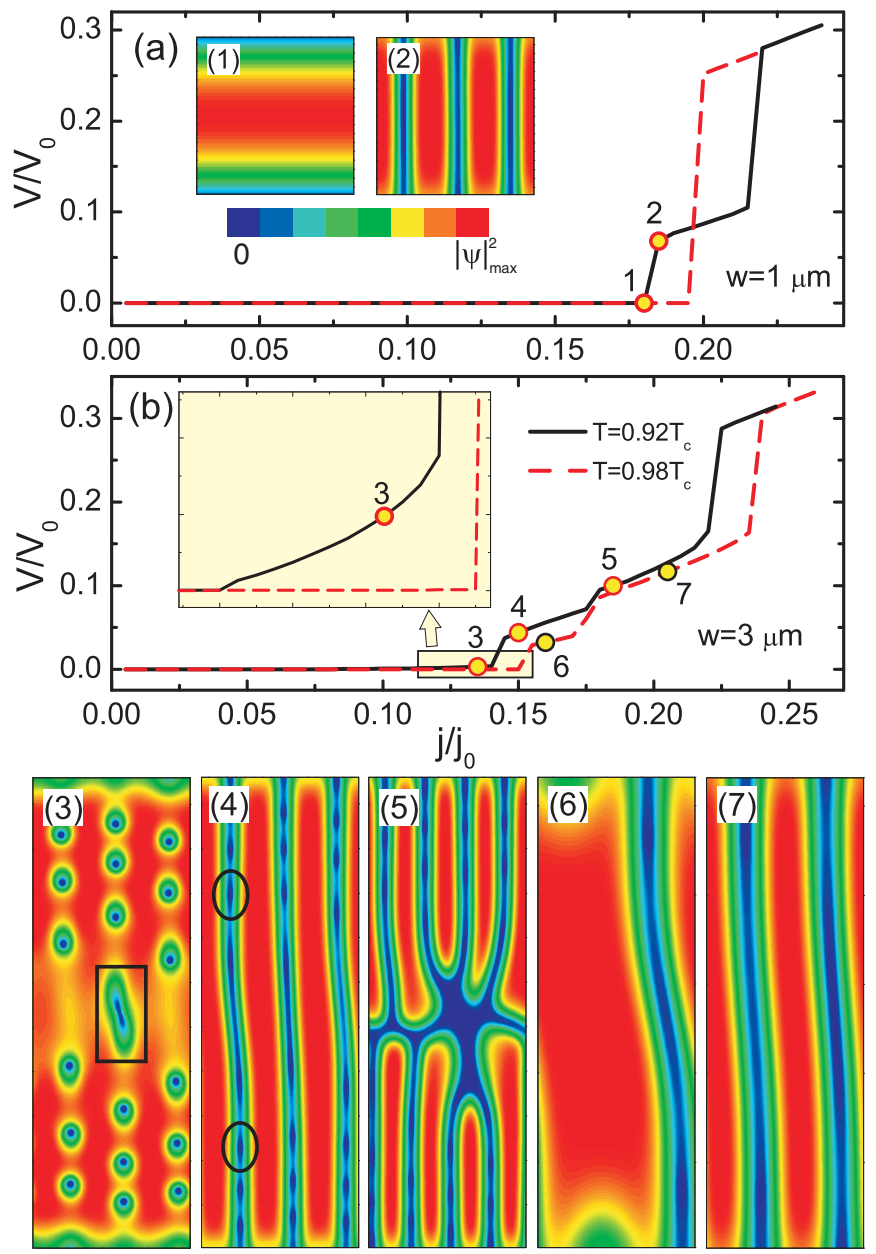

FIG. 2. (Color online) Time-averaged voltage vs current-density $(I-V)$ characteristics of the sample with width $w=1 \mu \mathrm{m}$ (a) and $w=3 \mu \mathrm{m}$ (b) at temperatures $T=0.92 T_{c}$ (solid black curve) and $T=0.98 T_{c}$ (dashed red curve). The length of the computational unit cell is $L=1 \mu \mathrm{m}$ and the GL parameter is $\kappa=10$. Inset in (b) shows the lower part of the $I-V$ curve. Panels 1-7 show snapshots of the Cooper-pair density $|\psi|^{2}$ (red/blue corresponds to largest/zero $|\psi|^{2}$ ) for current values indicated on the $I$ - $V$ curves. Black square in panel 3 shows the annihilation region of a vortex-antivortex pair and black circles in panel 4 indicate the positions of a fast-moving vortex (on top) and an antivortex (bottom).

$\xi(0)=10 \mathrm{~nm}$, which is the typical value for the $\mathrm{Nb}$ thin films (see, e.g., Ref. [22]). To investigate the response of the system to an external dc current, we first calculated the $I-V$ characteristics of the sample, which are shown in Fig. 2 for two values of the width $w$ and temperature. Since the voltage signal in our system is a time-dependent variable, we average the voltage over a time interval much larger than the characteristic voltage variation in order to construct the $I-V$ characteristics. For smaller systems [Fig. 2(a)] the zero resistance of the sample is maintained up to a threshold current $j_{c}$ [see point 1 and panel 1 in Fig. 2(a)], above which the system goes into the resistive state with a finite jump in the output voltage [point 2 in Fig. 2(a)]. This resistive state is characterized by fast-moving vortices [7,8,23,24]. With increasing temperature the system transits from the Meissner state directly into the normal state 
[see dashed curve in Fig. 2(a)] and no resistive state is observed during the current increase.

Figure 2(b) shows the $I-V$ characteristics of the sample with $w=3 \mu \mathrm{m}$. At lower temperatures, dissipation first arises due to the periodic nucleation and annihilation of slow-moving (Abrikosov) vortex-antivortex pairs in the center of the sample as shown in panel 3, where we plotted a snapshot of the Cooper-pair density. Since no external magnetic field is present, those vortices are created periodically at the opposite edges of the sample and annihilate in the middle of the sample (highlighted by a black square in panel 3). However, the voltage signal due to Abrikosov vortices is much smaller than the one generated by the fast-moving vortices [compare points 3 and 4 in Fig. 2(b)]. This signal increases monotonically with applied current indicating a larger nucleation rate of vortex-antivortex pairs. With increasing applied current, the triangular vortex lattice gets distorted and a rectangular lattice of vortices is observed (see panel 4) $[11,23]$. At larger current values, the vortices get deformed and resemble the structure of fast-moving (kinematic) vortices reported earlier $[7,8]$. The distortion is more pronounced in the middle of the sample during the annihilation of vortexantivortex pairs, where the speeds of the vortices are largest [8]. This transition from a slow to fast moving vortex state results in a noticeable jump in the $I$ - $V$ curve (see point 4 ). With further increase of the applied current more channels with fast-moving vortices appear in the system (see panel 5) before the entire sample transits into the normal state. The critical current density $j_{c}$ for the transition to the resistive state increases with increasing temperature (dashed red curve). However, the resistive state now is characterized only by fast-moving vortices (see panel 6). For a given value of the current density, the number of channels of fast-moving vortices (or the number of phase-slip lines) decreases with temperature (compare panels 5 and 7). The reason is that the effective size of the sample in units of the characteristic length scales [i.e., $\xi(T)$ and $\lambda(T)$ ] becomes smaller at high temperatures; thus it becomes difficult for the sample to accommodate more phase-slip lines. Note also that the unit for the voltage $V_{0}=c \Phi_{0} \rho_{n} / 8 \pi^{2} \lambda \xi$ explicitly contains temperature. Therefore, the voltage signal in real units is twice larger at $T=0.98 T_{c}$ as compared to the voltage measured at $T=0.9 T_{c}$. Thus, depending on the dimensions of the sample and on external conditions (i.e., current and temperature) the resistive state is characterized either by slow-moving Abrikosov vortices or fast-moving vortices (or phase-slip lines). Because of the small size of our sample, the formation of HSs leads to a transition to the normal state of the whole system.

\section{VOLTAGE-TIME CHARACTERISTICS}

Next, we study the response of our system to abrupt switching on of the applied current at zero external magnetic field, by constructing voltage vs time characteristics of the sample. For a given temperature and current, we started from the Meissner state (i.e., $|\psi|=1$ ) and applied a dc current pulse with duration $5000 t_{0}$ at $t=10 t_{0}$. During that time period, we recorded the voltage vs time $[V(t)]$ characteristics of the system together with the evolution of the superconduction state. To show the properties of the resistive state in response to the current we
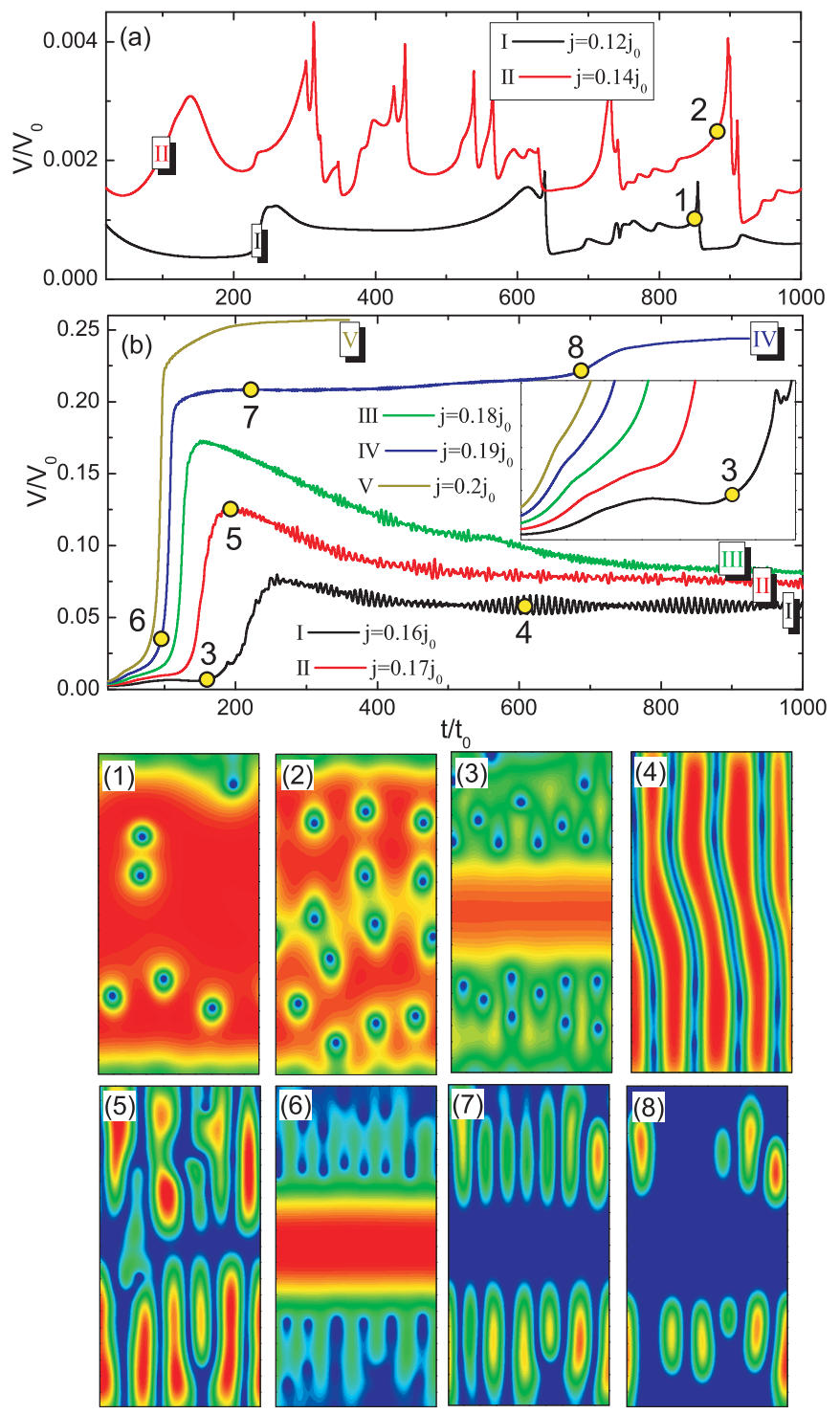

FIG. 3. (Color online) Voltage vs time characteristics of the sample with $L=1 \mu \mathrm{m}[L=31.6 \xi(T)]$ and $w=2 \mu \mathrm{m}[w=63.3 \xi(T)]$ at $T=0.9 T_{c}$ and for different values of the applied current $j \cdot u(t)$ $[u(t)$ is Heaviside unit step function], which is switched on abruptly. Panels 1-8 show snapshots of the Cooper-pair density $|\psi|^{2}$ [for the used scale see inset of Fig. 2(a)] at times indicated on the $V(t)$ curves. Inset in (b) shows a small time region of the $V(t)$ curves.

plot in Fig. $3 V(t)$ curves of the sample with dimensions $L=1$ $\mu \mathrm{m}$ and $w=2 \mu \mathrm{m}$ at $T=0.9 T_{c}$ for different values of the applied current density. Snapshots of the Cooper-pair density are also presented in order to show the time evolution of the superconducting condensate. At smaller currents [curves I and II in Fig. 3(a)] the output voltage oscillates in time with well defined maxima. Each maximum in the $V(t)$ curve corresponds either to a vortex penetration (see panel 1) or an annihilation of a vortex-antivortex pair (panel 2). However, the voltage signal is an order of magnitude smaller than the one we observed during the phase-slip line state [compare curves I in Figs. 3(a) and 3(b)]. Note that at these small time intervals, the dynamically stable state is not reached, where one observes periodic oscillations in $V(t)$ [22,25-29]. With 


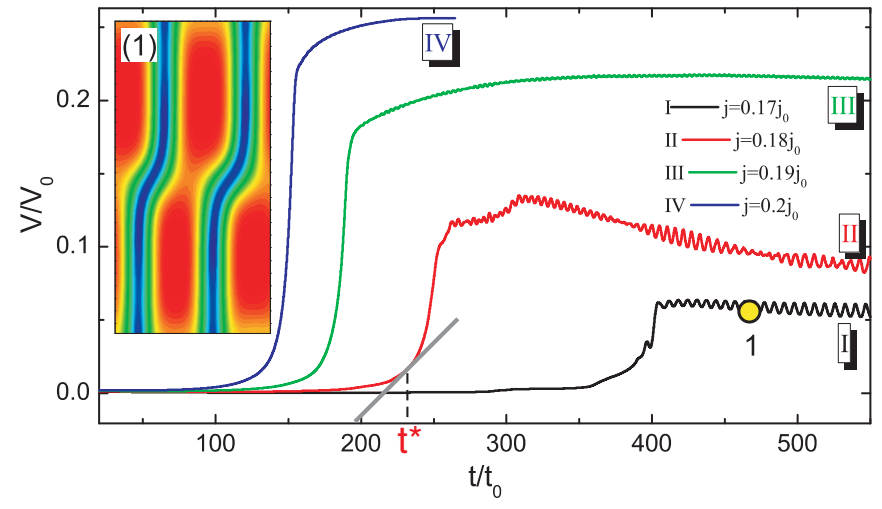

FIG. 4. (Color online) $V(t)$ curve of the sample with dimensions $L=1 \mu \mathrm{m}[L=20 \xi(T)]$ and $w=2 \mu \mathrm{m}[w=40 \xi(T)]$ at $T=$ $0.96 T_{c}$ and for different values of the applied current-density. Inset shows a snapshot of the Cooper-pair density $|\psi|^{2}$ at time indicated on the $V(t)$ curve.

increasing applied current, phase-slip lines are formed (panels 3 and 4) and the output voltage increases considerably [see curve I in Fig. 3(b)]. The average voltage signal saturates to a certain value with small oscillations, which are due to the penetration and/or annihilation of fast-moving vortices. With further increasing the applied current the nucleation rate of vortex-antivortex pairs, as well as the number of phase-slip lines, increases resulting in an increase of the output voltage. The voltage reaches a maxima just before the formation of phase-slip lines (point 5), which are due to nonequilibrium effects (see panel 5). However, the voltage curves saturate when the phase-slip state is established [see curves III and IV in Fig. 3(b)]. Please notice that the phase slip lines are not necessarily straight, but they are parallel to each other. With further increasing the applied current, the number and velocity of vortex-antivortex pairs increases (panel 6) and local hot spot areas appear, where the density of Cooper pairs reduces to zero. Those hot spots are created near the sample edges and in the middle of the sample (see panel 7), where the local velocity of the vortices become maximal (see Fig. 8 of Ref. [8]). Once created, the size of the hot spots increases in time (see panel 8 ) and the entire system becomes normal (point 8). For all the values of applied current and temperature, as well as for all considered sample sizes, the hot spots are unstable in time: they expand until the entire system makes a transition to the normal state.

Figure 4 shows the $V(t)$ curves of the sample considered in Fig. 3 but now for a higher temperature $T=0.96 T_{c}$ for different values of the current density. The resistive state here is also characterized by phase-slip lines (or kinematic vortices) at lower currents, which are stable in time (see the inset and curves I and II) and by hot spots at larger currents (curves III and IV), the formation of which results in the normal-state transition of the whole system. However, the number of phase slips is smaller than the one obtained at lower temperatures. With further increasing temperature, superconductivity exists only in the Meissner state and any motion of vortices results in a complete destruction of superconductivity (not shown here). This is because the sample dimensions become comparable to the characteristic length scales.
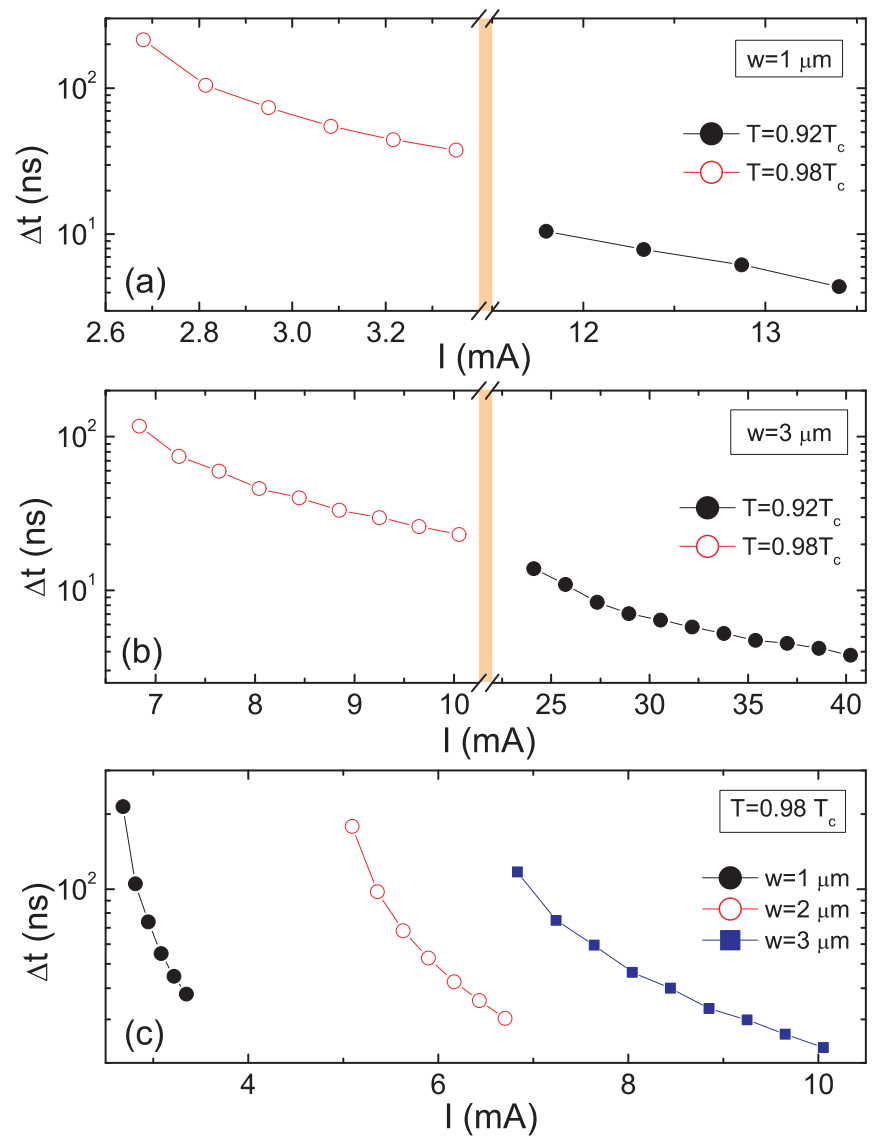

FIG. 5. (Color online) (a), (b) Delay time $\Delta t$ as a function of applied current at temperatures $T=0.92 T_{c}$ (filled black circles) and $T=0.98 T_{c}$ (open red circles) for the samples with width $w=1 \mu \mathrm{m}$ (a) and $w=3 \mu \mathrm{m}$ (b). (c) $\Delta t(I)$ curves of the samples with width $w=1 \mu \mathrm{m}$ (solid black circles), $w=2 \mu \mathrm{m}$ (open red circles), and $w=3 \mu \mathrm{m}$ (solid blue squares) at $T=0.98 T_{c}$. The length of the computational unit cell for all samples is $L=2 \mu \mathrm{m}$.

To summarize our findings on the response of superconducting strips to an external overcritical current, we plotted in Fig. 5 the delay time in the formation of the resistive state as a function of applied current for different width of the sample at different temperatures. The delay time is calculated as the difference between the switching time of the current pulse and the characteristic time $t^{*}$, when the slope of the $V(t)$ curve becomes $1 . t^{*}$ is indicated by the gray line in Fig. 4. The same method has been used to extract the delay time from the experimental results $[13,14]$. In converting the delay time and the current into real units we used the parameters of our $\mathrm{Nb}$ thin film samples (see Sec. V). Namely, the coherence length is $\xi(0)=10 \mathrm{~nm}$, penetration depth is $\lambda(0)=500 \mathrm{~nm}$, sample thickness is $d=80 \mathrm{~nm}$, and the normal-state resistivity $\rho=1.9 \times 10^{-7} \Omega \mathrm{m}$. For the parameters the time unit $t_{0}$ is around 100 ps near the critical temperature. It is seen from Fig. 5(a) that for all temperatures the response time monotonically decreases with increasing applied current. However, the transition to the resistive state occurs at larger currents when temperature is lowered [notice the break in the $x$ axis in Figs. 5(a) and 5(b)]. For a given value of the applied curve, the delay time becomes smaller 
at larger temperatures [compare solid black and open red curves in Fig. 5(b)]. All these findings are in good qualitative agreement with recent experiments on superconducting thin films [13-16]. Figure 5(c) shows the response time of all three investigated samples as a function of time at $T=0.98 T_{c}$. As expected, the delay increases with increasing size of the sample for given values of the applied current and temperature.

\section{SAMPLE AND EXPERIMENTAL SETUP}

As was shown in recent experiments in both low- and high-temperature superconductors [13,14], the phase-slip state initiates the hot-spot state at all temperatures. However, the phase-slip state is not always nucleated in single-pulse experiments: one observes the phase-slip state at temperatures larger than a characteristic temperature $T^{*}$, whereas the hot-spot state is realized for $T<T^{*}[13,14]$. This is due to the hysteretic behavior of superconductors. The phase-slip state for $T<T^{*}$ can be obtained only by implementing a sequence of stepped bias currents. However, such experiments are technologically very demanding and are not always accessible. Therefore, to investigate both phase-slip and hot-spot states, we studied several samples with the same dimensions, but with different $T^{*}$.

Niobium thin films grown by thermal co-evaporation were manufactured by the Cryo-electronics company (USA) on sapphire substrates with thickness of $d=80 \mathrm{~nm}$ and width $w=5 \mu \mathrm{m}$. We have selected two sample with the same transition temperatures $\left(T_{c}=8.7 \mathrm{~K}\right)$ and characteristic temperatures $T^{*}<4.2 \mathrm{~K}$ (sample 1) and $T^{*}>4.2 \mathrm{~K}$ (sample 2). The measurement consists of sending a single electrical pulse of $450 \mathrm{~ns}$ duration and $10 \mathrm{kHz}$ repetition through $50 \Omega$ coaxial cables. When the sample is in the superconducting state, it has no resistance. A delay line of $240 \mathrm{~ns}$ was used to separate the incident voltage from the reflected one at the sample stage. Incident and lateral voltages were measured using a fast numerical oscilloscope. The bias current through the sample was maintained constant by using a large resistance in series $R_{a}$, while a resistor $R_{\|}$is mounted in shunt across the combination $R_{a}+$ sample. The equivalent impedance at the line's termination is $50 \Omega$, and as a consequence the reflected pulse vanishes. For an incident voltage $V_{i}$, the current flowing through the strip in its superconducting state is $I=$ $I_{\mathrm{Tot}} R_{\|} /\left(R_{\|}+R_{a}\right)$, where the circuit impedance $Z=50 \Omega$ and $I_{\mathrm{Tot}}=\frac{V_{i}}{Z}$. The voltage response was recorded using a fast oscilloscope through lateral electrodes with $187 \Omega$ connected in series [Fig. 6(b)].

\section{PSC AND HS CREATED BY A CURRENT PULSE}

The sample response to an electrical pulse was measured by increasing the current pulse amplitude; different dissipative modes occurred depending on the applied current and substrate temperature $T_{b}$. When a current pulse $I \geqslant I_{c}$ was sent to the sample, a voltage response delay appeared with respect to the current. Close to $T_{c}$, usually a flux flow (FF) response starting at time $t=0$ without any delay time $t_{d}$ was observed due to the motion of vortices. The FF voltage can be reduced by lowering the substrate temperature. Another dissipative mode occurred when the current exceeds the critical current;

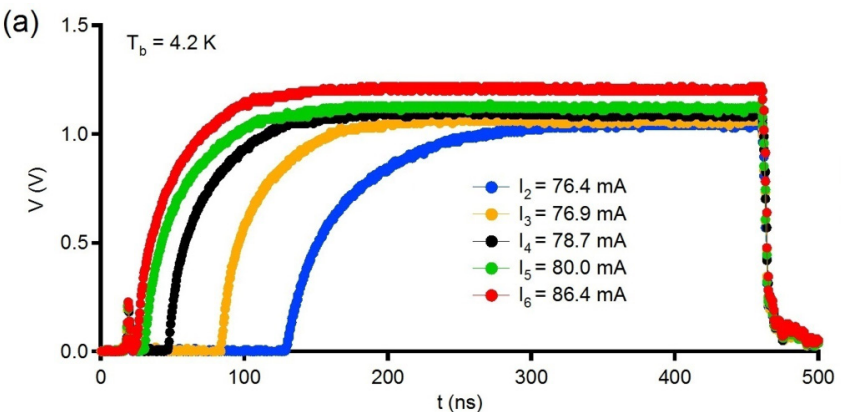

(b)

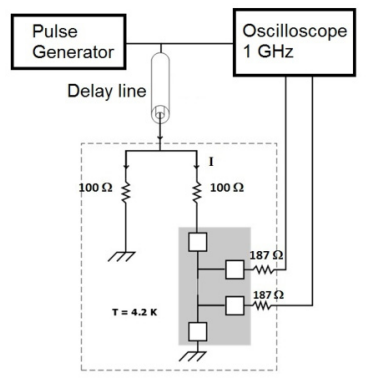

(c)

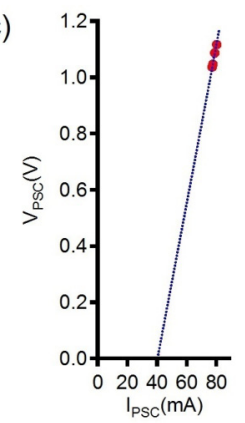

FIG. 6. (Color online) (a) Voltage response of $\mathrm{Nb}$ sample 1 to current pulses as a function of time at $T_{b}=4.2 \mathrm{~K}$. The critical current is $I_{c}(4.2 \mathrm{~K})=72.2 \mathrm{~mA}$. (b) Schematics of the experimental setup $\left(R_{\|}=R_{\perp}=100 \Omega\right.$ ). (c) PSC plateau voltage as a function of the applied current. The superconducting excess current is $I_{s}=40 \mathrm{~mA}$.

a PSC is nucleated or a hot spot is formed $[1,5,30] . I_{c}$ is the minimum critical current to nucleate a PSC or to destroy locally superconductivity.

A PSC can be distinguished from a hot spot by its voltage response. Both modes are characterized by a noticeable increase in voltage after a delay time $t_{d}$. The delay time $t_{d}$ is described by the following TDGL time [30]:

$$
t_{d}\left(I / I_{c}\right)=\tau_{d} \int_{0}^{1} \frac{2 f^{4} d f}{\frac{4}{27}\left(\frac{I}{I_{c}}\right)^{2}-f^{4}+f^{6}} .
$$

The PSC is characterized by a constant-in-time voltage, whereas the hot spot shows a linear variation of the voltage as a function of time [see the discussion related to Fig. 3(b)]. The temperature inside the PSC is lower than the critical one (i.e., $T_{\mathrm{PSC}}<T_{c}$ ), while the temperature inside the hot spots exceeds the critical value $T_{\mathrm{HS}}>T_{c}$, destroying superconductivity completely.

Figure 6(a) shows the $V(t)$ curves of sample 1 at $T=4.2 \mathrm{~K}$ for different values of the applied current. It is seen from this figure that the response time of the sample to the abrupt switching on of the current decreases with increasing current value. Such a behavior of the delay time is also found in our numerical simulations (see Fig. 5). After this characteristic time, the voltage signal increases with time until it saturates to a certain value. This phenomenon, already reported in low$T_{c}$ [13] and high- $T_{c}$ [14] superconductors, was related to the formation of a PSC. Our numerical simulations also confirm that (see Fig. 3 and corresponding discussions) the phase-slip state is characterized by the saturation of the output voltage. Increasing the applied current results in an increase in the output 


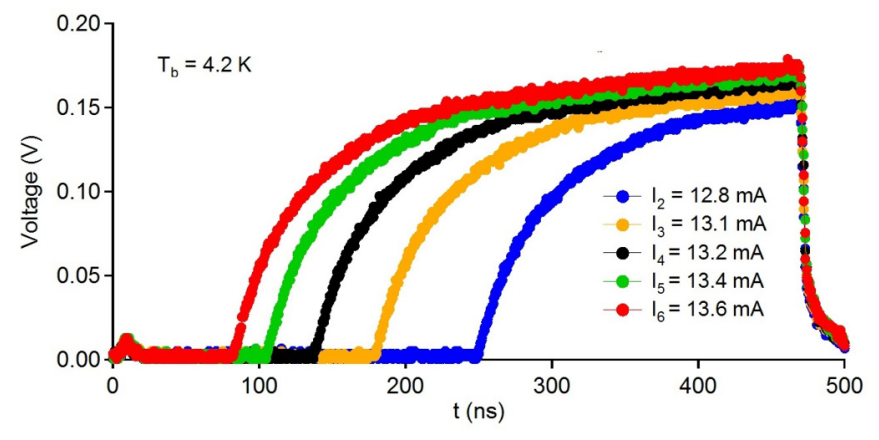

FIG. 7. (Color online) Voltage versus time across $\mathrm{Nb}$ sample 2 for 5 current pulse amplitudes. The nonsaturation of $V(t)$ after several hundred ns indicate a slowly growing hot spot, in contrast to the PSCs of Fig. 6. Note the gradual decrease of the delay time with the current, similarly to the PSC delay times in Fig. 6.

voltage without expansion of the PSC's length. The differential resistance of the PSC is extracted from the linear dependence of the PSC's voltage vs the applied current. As was predicted by the PSC theory [5], the extrapolated slope of VPSC vs IPSC curve intercepts the current axis at the excess superconducting excess current $I_{s}$, which in our case equals $40 \mathrm{~mA}$ [see Fig. 6(c)]. The PSC was interpreted as an oscillation of the order parameter between 0 and 1 . Therefore, the total current is the sum of the two currents, the normal and superconducting currents. The heat dissipated in this localized zone per unit volume is $\rho I\left(I-I_{s}\right) /(w d)^{2}$, where $\rho$ and $I_{s}$ are respectively the normal resistivity and the superconducting current [1].

Figure 7 shows the $V(t)$ curves of sample 2 at $T=4.2 \mathrm{~K}$ for different values of the applied current. Although this sample has the same dimensions as sample 1, it shows a slightly different response to the applied current. The output voltage increases rapidly after the delay time which we relate to the formation of PSCs. This process is followed by a slow variation of the $V(t)$ curve. By increasing the applied current the delay time is reduced, the voltage increases, and the slope increases corresponding to the expansion velocity of the HS along the film. The normal spot expands and the current is purely normal. The heat generated per unit volume is $\rho I^{2} /(w b)^{2}$ and escapes towards the substrate. The transformation of the PSC to HS is obvious from these results: the voltage curves do not saturate. This is due to the fact that the bath temperature is larger than $T^{*}$. Similar behavior of $V(t)$ is shown in Fig. 3(b) by curves IV and V. On increasing the bath temperature $T_{b}$, a more abrupt decrease of $I_{c}$ as compared to that of $I_{h}$ reverses the situation, and thus places $I_{c}\left(T_{b}\right)$ below $I_{h}\left(T_{b}\right)$ [14], where $I_{h}$ is the minimum current, whose Joule effect is sufficient to maintain a well defined normal spot above $T_{c}$.

By comparing theoretical (Figs. 3-5) and experimental (Figs. 6 and 7) results, we find the following agreements between theory and experiment: (i) the output voltage signal saturates with time when the system is in the phase-slip state, (ii) a monotonic increase of the voltage is observed when the system is in the hot-spot state, and (iii) the response time of the system decreases monotonically with temperature and applied current. However, the theoretical estimate for the delay time is smaller than the experimentally obtained value, which is due to the smaller size of the sample studied theoretically.

\section{CONCLUSIONS}

Using the time-dependent Ginzburg-Landau theory and pulsed-current measurements, we studied the response of a superconducting thin-film sample to abrupt switching on of external current. Simulation results showed that depending on the value of the applied current and/or temperature, the resistive state is characterized by either (i) the flux-flow state, (ii) the phase-slip state (or fast-moving vortices), or (iii) the hot-spot state. The first two states are found to be dynamically stable in time, whereas the hot-spot state is characterized by the spread of the normal-state region until the whole sample switches to the normal state. The latter regime results in a linear increase of the output voltage with time. The characteristic time for the transition to the resistive state (i.e., the delay time) decreases by increasing the applied current at fixed temperature or by increasing the temperature for a given value of the applied current in agreement with experiment. Our theoretical findings are in good qualitative agreement with our experiments on $\mathrm{Nb}$ strips. The different dissipation modes induced by the supercritical current were discriminated in the experimental results, and the phase-slip and hot-spot regimes were clearly identified.

\section{ACKNOWLEDGMENTS}

This work was supported by EU Marie Curie Project No. 253057, the Flemish Science Foundation (FWO-V1), and King Fahd University of Petroleum and Minerals, Saudi Arabia, under the IN131034 DSR project.
[1] M. Tinkham, Introduction to Superconductivity, 2nd ed. (McGraw-Hill, Singapore, 1996), Chap. 11.

[2] B. I. Ivlev and N. B. Kopnin, Usp. Fiz. Nauk 142, 435 (1984); Sov. Phys. Usp. 27, 206 (1984).

[3] W. W. Webb and R. J. Warburton, Phys. Rev. Lett. 20, 461 (1968).

[4] J. D. Meyer, App. Phys. 2, 303 (1973).

[5] W. J. Skocpol, M. R. Beasley, and M. Tinkham, J. Low Temp. Phys. 16, 145 (1974).
[6] A. Weber and L. Kramer, J. Low Temp. Phys. 84, 289 (1991).

[7] A. Andronov, I. Gordion, V. Kurin, I. Nefedov, and I. Shereshevsky, Physica C 213, 193 (1993).

[8] G. R. Berdiyorov, M. V. Milošević, and F. M. Peeters, Phys. Rev. B 79, 184506 (2009).

[9] A. G. Sivakov, A. M. Glukhov, A. N. Omelyanchouk, Y. Koval, P. Muller, and A. V. Ustinov, Phys. Rev. Lett. 91, 267001 (2003).

[10] M. Tinkham, J. Low Temp. Phys. 35, 147 (1979). 
[11] D. Y. Vodolazov and F. M. Peeters, Phys. Rev. B 76, 014521 (2007).

[12] W. J. Skocpol, M. R. Beasley, and M. Tinkham, J. Appl. Phys. 45, 4054 (1974).

[13] F.-R. Ladan, Kh. Harrabi, M. Rosticher, C. Villard, P. Mathieu, and J.-P. Maneval, J. Low Temp. Phys. 153, 103 (2008).

[14] K. Harrabi, F. R. Ladan, Vu Dinh Lam, J.-P. Maneval, J.-F. Hamet, J.-P. Villégier, and R. W. Bland, J. Low Temp. Phys. 157, 36 (2009).

[15] K. Harrabi, J. Supercond. Nov. Magn. 26, 1865 (2013).

[16] J. P. Maneval, K. Harrabi, F. Chibane, M. Rosticher, F. R. Ladan, and P. Mathieu, IEEE Trans. Appl. Supercond. 23, 2200604 (2013).

[17] G. J. Dolan and L. D. Jackel, Phys. Rev. Lett. 39, 1628 (1977).

[18] R. B. Laibowitz, A. N. Broers, J. T. C. Yeh, and J. M. Viggiano, App. Phys. Lett. 35, 891 (1979).

[19] L. Kramer and R. J. Watts-Tobin, Phys. Rev. Lett. 40, 1041 (1978); R. J. Watts-Tobin, Y. Krähenbühl, and L. Kramer, J. Low Temp. Phys. 42, 459 (1981).

[20] T. Winiecki and C. S. Adams, J. Comput. Phys. 179, 127 (2002).

[21] D. Y. Vodolazov, F. M. Peeters, M. Morelle, and V. V. Moshchalkov, Phys. Rev. B 71, 184502 (2005).
[22] G. R. Berdiyorov, M. V. Milosevic, M. L. Latimer, Z. L. Xiao, W. K. Kwok, and F. M. Peeters, Phys. Rev. Lett. 109, 057004 (2012).

[23] A. V. Silhanek, M. V. M. V. Milošević, R. B. G. Kramer, G. R. Berdiyorov, J. Van de Vondel, R. F. Luccas, T. Puig, F. M. Peeters, and V. V. Moshchalkov, Phys. Rev. Lett. 104, 017001 (2010).

[24] L. N. Bulaevskii, M. J. Graf, and V. G. Kogan, Phys. Rev. B 85, 014505 (2012).

[25] G. R. Berdiyorov, A. K. Elmurodov, F. M. Peeters, and D. Y. Vodolazov, Phys. Rev. B 79, 174506 (2009).

[26] G. R. Berdiyorov, M. V. Milošević, and F. M. Peeters, Phys. Rev. B 80, 214509 (2009).

[27] G. R. Berdiyorov, X. H. Chao, F. M. Peeters, H. B. Wang, V. V. Moshchalkov, and B. Y. Zhu, Phys. Rev. B 86, 224504 (2012).

[28] J. R. Clem, Y. Mawatari, G. R. Berdiyorov, and F. M. Peeters, Phys. Rev. B 85, 144511 (2012).

[29] G. R. Berdiyorov, A. R. de C. Romaguera, M. V. Milošević, M. M. Doria, L. Covaci, and F. M. Peeters, Eur. Phys. J. B 85, 130 (2012).

[30] J. A. Pals and J. Wolter, Phys. Lett. A 70, 150 (1979). 